\title{
Noncommutativity due to spin
}

\author{
M.Gomes, V.G. Kupriyanov†, A.J. da Silva ${ }^{\ddagger}$ \\ Instituto de Física, Universidade de São Paulo, Brazil
}

August 18, 2021

\begin{abstract}
Using the Berezin-Marinov pseudoclassical formulation of spin particle we propose a classical model of spin noncommutativity. In the nonrelativistic case, the Poisson brackets between the coordinates are proportional to the spin angular momentum. The quantization of the model leads to the noncommutativity with mixed spacial and spin degrees of freedom. A modified Pauli equation, describing a spin half particle in an external e.m. field is obtained. We show that nonlocality caused by the spin noncommutativity depends on the spin of the particle; for spin zero, nonlocality does not appear, for spin half, $\Delta x \Delta y \geq \theta^{2} / 2$, etc. In the relativistic case the noncommutative Dirac equation was derived. For that we introduce a new star product. The advantage of our model is that in spite of the presence of noncommutativity and nonlocality, it is Lorentz invariant. Also, in the quasiclassical approximation it gives noncommutativity with a nilpotent parameter.
\end{abstract}

\section{Introduction}

The idea of using noncommutative (NC) coordinates in quantum mechanics appeared a long time ago. In [1] noncommutative coordinates were used to describe the charged particle in the strong magnetic field and in the presence of the weak electric potencial. In the last decade, remotivated by string theory arguments [2], the subject gained a lot of interest and has been studied extensively (see e.g. [3] and [4] for reviews on noncommutativity in QFT and QM, respectively). The canonical noncommutative space can be realized by the coordinate operators $\hat{x}^{i}$, satisfying commutation relations $\left[\hat{x}^{i}, \hat{x}^{j}\right]=i \theta^{i j}$, where $\theta^{i j}$ is an antisymmetric constant matrix.

\footnotetext{
*e-mail: mgomes@fma.if.usp.br

†e-mail: vladislav.kupriyanov@gmail.com

${ }^{\ddagger}$ e-mail: ajsilva@fma.if.usp.br
} 
Recently, other types of noncommutativity, different from the canonical, have also been considered. Thus, in [5] it was proposed a model of position dependent noncommutativity in quantum mechanics. In [6] a model of dynamical noncommutativity was discussed. The authors of [7] have proposed a three-dimensional noncommutative quantum mechanical system with mixing spacial and spin degrees of freedom. The noncommutative spatial coordinates $\hat{x}^{i}$, the conjugate momenta $\hat{p}_{i}$, and the spin variables $\hat{s}^{i}$ were supposed to satisfy the non-standard Heisenberg algebra:

$$
\begin{aligned}
& {\left[\hat{x}^{i}, \hat{x}^{j}\right]=i \theta^{2} \varepsilon^{i j k} \hat{s}^{k},} \\
& {\left[\hat{x}^{i}, \hat{p}_{j}\right]=i \delta_{j}^{i}, \quad\left[\hat{p}_{i}, \hat{p}_{j}\right]=0,} \\
& {\left[\hat{x}^{i}, \hat{s}^{j}\right]=i \theta \varepsilon^{i j k} \hat{s}^{k}, \quad\left[\hat{s}^{i}, \hat{s}^{j}\right]=i \varepsilon^{i j k} \hat{s}^{k},}
\end{aligned}
$$

where $\theta$ is the parameter of noncommutativity (a real number). We will call it spin noncommutativity. Later, in [8] it was elaborated an approach to the BoseEinstein condensation theory, based on the spin noncommutativity. Note that in $2+1$ dimensions the relation between anyon spin and noncommutativity was discussed in [9].

In the present work we will discuss some questions regarding the physical meaning and mathematical formulation of the spin noncommutativity (11).

It is known that canonical NCQM [4] can be obtained as a result of quantization of classical models, see e.g., [10]- [12]. The corresponding action functional appears as an effective action in path integral representation of NCQM [13]- [15] and can be used for study of global and local symmetries of the system [16], etc. The first question is if there exists a classical model, which after quantization lead to the spin noncommutativity.

Another question is connected with nonlocality. Usually, noncommutativity means the presence of nonlocality, i.e., nontrivial uncertainty relations between the coordinates,

$$
\Delta x^{i} \Delta x^{j} \geq \text { something } \neq 0 .
$$

The question is what is the form of nonlocality caused by spin noncommutativity? Also, it is important to understand how the presence of the spin noncommutativity can affect the relations between spin and statistics; however, we will not discuss it in the present paper.

The last point, we would like to discuss here, is how to formulate a consistent relativistic version of spin noncommutativity. In particular, we will obtain the modification of the Dirac equation in the case of spin noncommutativity.

The paper is organized as follows. In the Sec. 2 we discuss the classical model. The quantization of this model, constructed in Sec. 3, leads to the modified Pauli equation and not to the Schrodinger one. We also discuss the possibility of relativistic generalization of our model. 


\section{Particle spin dynamics and its noncommuta- tive deformation}

In [17] Berezin and Marinov have proposed a classical model of the spin $1 / 2$ particle, involving Grassmann degrees of freedom. In the nonrelativistic case, the classical mechanics of a particle with spin is constructed in the phase superspace, consisting of the six-dimmensional orbital subspace $\left(x^{i}, p_{i}\right), i=1,2,3$, and threedimensional spin Grassmann subspace $\xi^{i}, \xi^{i} \xi^{j}+\xi^{j} \xi^{i}=0$.

The Poisson bracket between two arbitrary functions $f$ and $g$ of the Grassmann variables is determined as follows,

$$
\{f(\xi), g(\xi)\}=-i\left(f \overleftarrow{\partial_{k}}\right)\left(\overrightarrow{\partial_{k}} g\right)
$$

This Poisson bracket is antisymmetric if both functions are even elements of the Grassmann algebra, and if one of them is an even element while other one is an odd element. If both functions are odd elements, the Poisson bracket is symmetric. For the canonical variables, the Poisson brackets are

$$
\left\{\xi^{k}, \xi^{l}\right\}=-i \delta^{k l}, \quad\left\{x^{k}, p_{l}\right\}=\delta_{l}^{k}
$$

The rotation group in the Grassmann subspace is generated by the spin angular momentum

$$
\begin{aligned}
& S^{i}=-\frac{i}{2} \varepsilon^{i j k} \xi^{j} \xi^{k}, \\
& \left\{S^{i}, \xi^{j}\right\}=\varepsilon^{i j k} \xi^{k}, \quad\left\{S^{i}, S^{j}\right\}=\varepsilon^{i j k} S^{k} .
\end{aligned}
$$

The orbital angular momentum $L^{i}=\varepsilon^{i k l} x^{k} p^{l}$ generates the rotation group in the orbital subspace,

$$
\left\{L^{i}, x^{j}\right\}=\varepsilon^{i j k} x^{k}, \quad\left\{L^{i}, L^{j}\right\}=\varepsilon^{i j k} L^{k} .
$$

The complete angular momentum is determined as being

$$
\mathbf{J}=\mathbf{L}+\mathbf{S}, \quad\left\{J^{i}, J^{j}\right\}=\varepsilon^{i j k} J^{k} .
$$

The classical Hamiltonian action of the model reads

$$
S_{0}=\int d t\left[\mathbf{p} \dot{\mathbf{x}}-\frac{i}{2} \xi \dot{\xi}-H(x, p, \xi)\right]
$$

where

$$
H(x, p, \xi)=\frac{\mathbf{p}^{2}}{2}+V_{0}(x)+(\mathbf{L S}) V_{1}(x)+\mathbf{S B}(x),
$$

\footnotetext{
${ }^{1}$ The similar model was considered independently in [18].
} 
$V_{0}(x)$ and $V_{1}(x)$ are potential functions, and $\mathbf{B}(x)$ is a vector field. The term with $V_{1}$ in (5) is the spin-orbit interaction. The quantization of the theory (5) leads to the Pauli equation describing quantum nonrelativistic spin $1 / 2$ particle.

Now, let us deform the above model to obtain nonzero Poisson brackets between the coordinates, which may lead to noncommutativity after quantization. The simplest way to do it is to mix coordinates and momenta [12], $x_{N C}^{i}=x^{i}-1 / 2 \theta^{i j} p_{j}$. However, this breaks symmetries of the system, e.g., rotational symmetry, as $x_{N C}^{i}$ is not a vector anymore (it does not transform as a vector, since $\theta^{i j}$ is a constant matrix). To preserve rotational symmetry, one can mix coordinates and spin angular momentum:

$$
\tilde{x}^{i}=x^{i}+\theta S^{i} .
$$

These new coordinates $\tilde{x}^{i}$, like the old ones are even and transform like a vector,

$$
\left\{J^{i}, \tilde{x}^{j}\right\}=\varepsilon^{i j k} \tilde{x}^{k} .
$$

The nonvanishing Poisson brackets, involving new coordinates, are

$$
\begin{aligned}
& \left\{\tilde{x}^{i}, \tilde{x}^{j}\right\}=\theta^{2} \varepsilon^{i j k} S^{k}, \quad\left\{\tilde{x}^{i}, p_{j}\right\}=\delta_{j}^{i}, \\
& \left\{\tilde{x}^{i}, \xi^{j}\right\}=\theta \varepsilon^{i j k} \xi^{k}, \quad\left\{\xi^{k}, \xi^{l}\right\}=-i \delta^{k l} .
\end{aligned}
$$

Let us suppose that $\tilde{x}^{i}$ are "physical", i.e., observable coordinates. We note that the center of mass coordinates in the Schrodinger Zitterbewegung problem satisfy similar commutation relations as $\tilde{x}^{i}$, see [19]. One can then treat Poisson brackets (91) as fundamental Poisson brackets of a new theory in a phase superspace $(\tilde{x}, p, \xi)$. The graded version of the Jacobi identity in the deformed theory can be easily verified. The Hamiltonian of the deformed theory is $H(\tilde{x}, p, \xi)$, where $H(x, p, \xi)$ was determined in (6) .

In fact, this deformation is equivalent to the addition of new terms in the action (5), which disappear in the limit $\theta \rightarrow 0$. However, since we already have a consistent Hamiltonian formulation, which is necessary for the quantization, the exact form of these additional terms is immaterial 2

\section{Quantization}

In the course of quantization we replace the Poisson brackets (91) between the canonical variables by the commutator (anticommutator) of the corresponding operators

$$
\begin{aligned}
& {\left[\hat{x}^{i}, \hat{x}^{j}\right]=i \theta^{2} \varepsilon^{i j k} \hat{s}^{k}, \quad\left[\hat{x}^{i}, \hat{p}_{j}\right]=i \delta_{j}^{i},} \\
& {\left[\hat{x}^{i}, \hat{\xi}^{j}\right]=i \theta \varepsilon^{i j k} \hat{\xi}^{k}, \quad\left[\hat{\xi}^{i}, \hat{\xi}^{j}\right]_{+}=\delta^{i j} .}
\end{aligned}
$$

\footnotetext{
${ }^{2}$ The corresponding action functional can be constructed along the lines described in 20 , taking into account the presence of the Grassmann variables.
} 
Renormalizing the operators $\hat{\xi}^{i}=\hat{\sigma}^{i} / \sqrt{2}$, one gets the Clifford algebra with three generators

$$
\left[\hat{\sigma}^{i}, \hat{\sigma}^{j}\right]_{+}=2 \delta^{i j}
$$

The only irreducible representation of this algebra is two-dimensional, it can be realized by the Pauli matrices $\sigma^{i}$. Consequently,

$$
\hat{s}^{i}=-\frac{i}{2} \varepsilon^{i j k} \hat{\xi}^{j} \hat{\xi}^{k}=\frac{1}{2} \sigma^{i} .
$$

One can see that the commutation relations involving the spatial coordinates $\hat{x}^{i}$, the conjugate momenta $\hat{p}_{i}$, and the spin variables $\hat{s}^{i}$ are exactly those in (1), as postulated in [7]. However, we have obtained these commutation relations as result of a consistent quantization of a corresponding classical theory.

The representation of the quantum algebra (1) is

$$
\hat{x}^{i}=x^{i} \mathbf{I}+\theta \hat{s}^{i}, \quad \hat{p}_{i}=-i \partial_{i} \mathbf{I},
$$

where $\mathbf{I}$ is the $2 \times 2$ unit matrix, and $\hat{s}^{i}$ are determined in (12). The modified Pauli equation, describing a nonrelativistic spinning particle in an external electromagnetic field is

$$
i \partial_{t} \varphi=\hat{H}(\hat{x}, \hat{p}, \hat{\xi}) \varphi
$$

where $\varphi$ is a Pauli spinor and the Hamiltonian is given in (66).

According to (11) one has the uncertainty relations

$$
\Delta x^{i} \Delta x^{j} \geq \theta^{2} \varepsilon^{i j k}\left|\left\langle\Psi\left|\hat{s}^{k}\right| \Psi\right\rangle\right|
$$

where $|\Psi\rangle$ is a given state. Note, that since the operators $\hat{s}^{k}$ do not commute, one can not measure simultaneously eigenvalues for all operators $\hat{s}^{k}$, one has to choose one of them, e.g., $\hat{s}_{z}$. If the particle has spin zero, then $\hat{s}^{k}|\Psi\rangle=0$, there is no nonlocality in this case. For the spin $s$ different from zero, one has:

$$
\hat{s}_{z}|\Psi\rangle=s_{z}|\Psi\rangle, \quad s_{z}=-s,-s+1, \ldots, s .
$$

Substituting this in (15) one has

$$
\Delta x \Delta y \geq \theta^{2}\left|s_{z}\right|
$$

For the particle with the spin $s=1 / 2$,

$$
\Delta x \Delta y \geq \frac{\theta^{2}}{2}
$$

So, for the spin noncommutativity, nonlocality is proportional to the quantum number $s_{z}$, i.e., depends on the spin of the particle. Physically one can interpret this result as follows: the maximal precision to localize the particle depends on its spin. 


\section{Relativistic generalization}

In the relativistic case, the Hamiltonian form of the Berezin-Marinov action is

$$
\begin{aligned}
& S=\int_{\tau_{i}}^{\tau_{f}}\left[p_{\mu} \dot{x}^{\mu}-\frac{i}{2} \xi_{\mu} \dot{\xi}^{\mu}+\frac{i}{2} \xi^{5} \dot{\xi}^{5}-\frac{i}{2} \chi T_{1}-\lambda T_{2}\right] d \tau \\
& T_{1}=\xi^{\mu}\left(p_{\mu}+e A_{\mu}\right)+m \xi^{5}, \quad T_{2}=\left(p_{\mu}+e A_{\mu}\right)^{2}-m^{2}+i e F_{\mu \nu} \xi^{\mu} \xi^{\nu},
\end{aligned}
$$

here $\xi^{\mu}, \xi^{5}$ are Grassmann variables, describing spin degrees of freedom, $\lambda$ and $\chi$ are Lagrange multipliers, $\lambda$-commuting and $\chi$-anticommuting. Nonvanishing Poisson brackets between the canonical variables are

$$
\left\{x^{\mu}, p^{\nu}\right\}=g^{\mu \nu}, \quad\left\{\xi^{\mu}, \xi^{\nu}\right\}=-i g^{\mu \nu}, \quad\left\{\xi^{5}, \xi^{5}\right\}=i
$$

where $g^{\mu \nu}=\operatorname{diag}(1,-1,-1,-1)$. Also, one has two first-class constraints:

$$
T_{1}=0, \quad T_{2}=0
$$

Observe that $T_{1}$ is an odd element of the Grassmann algebra, therefore the Poisson bracket of $T_{1}$ with $T_{1}$ is not zero, but

$$
\left\{T_{1}, T_{1}\right\}=-i T_{2}
$$

By its definition, $T_{2}$ is even, so that $\left\{T_{2}, T_{2}\right\}=0$, and

$$
\left\{T_{2}, T_{1}\right\}=i\left\{\left\{T_{1}, T_{1}\right\}, T_{1}\right\} \equiv 0
$$

due to the Jacobi identity. Thus, we have proved that (18) are indeed first-class constraints.

Generators of the Lorentz group $J_{\mu \nu}$ are defined as

$$
\begin{aligned}
& J^{\mu \nu}=L^{\mu \nu}+S^{\mu \nu}, \\
& L^{\mu \nu}=x^{\mu} p^{\nu}-x^{\nu} p^{\mu}, \quad S^{\mu \nu}=-i \xi^{\mu} \xi^{\nu} .
\end{aligned}
$$

In the classical theory

$$
\begin{aligned}
& \left\{L^{\mu \nu}, x^{\lambda}\right\}=g^{\mu \lambda} x^{\nu}-g^{\nu \lambda} x^{\mu} \\
& \left\{S^{\mu \nu}, \xi^{\lambda}\right\}=g^{\mu \lambda} \xi^{\nu}-g^{\nu \lambda} \xi^{\mu}
\end{aligned}
$$

To construct relativistic generalization of the spin type noncommutativity we introduce new coordinates

$$
\tilde{x}^{\mu}=x^{\mu}+\theta W^{\mu},
$$

where

$$
W^{\mu}=\frac{1}{2} \varepsilon^{\mu \nu \rho \sigma} p_{\nu} J_{\rho \sigma}=\frac{1}{2} \varepsilon^{\mu \nu \rho \sigma} p_{\nu} S_{\rho \sigma}
$$


is the Pauli-Lubanski vector. By the definition, $\tilde{x}^{\mu}$ is an even element of the Grassmann algebra, and it transforms like a vector,

$$
\left\{J^{\mu \nu}, \tilde{x}^{\lambda}\right\}=g^{\mu \lambda} \tilde{x}^{\nu}-g^{\nu \lambda} \tilde{x}^{\mu} .
$$

The Poisson brackets involving new coordinates are

$$
\begin{aligned}
& \left\{\tilde{x}^{\mu}, \tilde{x}^{\nu}\right\}=-\theta \varepsilon^{\mu \nu \rho \sigma} S_{\rho \sigma}-\frac{\theta^{2}}{2} \varepsilon^{\mu \nu \rho \sigma} W_{\rho} p_{\sigma}, \\
& \left\{\tilde{x}^{\mu}, p^{\nu}\right\}=g^{\mu \nu}, \quad\left\{\xi^{\mu}, \xi^{\nu}\right\}=-i g^{\mu \nu}, \quad\left\{\xi^{5}, \xi^{5}\right\}=i, \\
& \left\{\tilde{x}^{\mu}, \xi^{\nu}\right\}=-\theta \varepsilon^{\mu \nu \rho \sigma} p_{\rho} \xi_{\sigma} .
\end{aligned}
$$

Again, we treat coordinates $\tilde{x}^{\mu}$ as "physical" coordinates and Poisson brackets (25) as the fundamental Poisson brackets of a new theory in a phase superspace $(\tilde{x}, p, \xi)$. The constraints (18) should be modified. We postulate the form of the first constraint as

$$
\tilde{T}_{1}=\xi^{\mu}\left(p_{\mu}+e A_{\mu}(\tilde{x})\right)+m \xi^{5}=0 .
$$

As in undeformed case, it is an odd element of the Grassmann algebra, since $\tilde{x}^{\mu}$ is even. Following (19) we determine the second constraint as

$$
\begin{aligned}
& \tilde{T}_{2}=i\left\{\tilde{T}_{1}, \tilde{T}_{1}\right\}=0, \\
& \tilde{T}_{2}=\left(p_{\mu}+e A_{\mu}\right)^{2}-m^{2}+i e \tilde{F}_{\mu \nu} \xi^{\mu} \xi^{\nu}+2 i e\left\{\xi^{\mu}, A_{\nu}\right\}\left(p_{\mu}+e A_{\mu}\right) \xi^{\nu}, \\
& \tilde{F}_{\mu \nu}=\frac{1}{e}\left\{p_{\mu}+e A_{\mu}(\tilde{x}), p_{\nu}+e A_{\nu}(\tilde{x})\right\} .
\end{aligned}
$$

It is even, since the Poisson bracket of two odd elements is always even. Therefore, $\left\{\tilde{T}_{2}, \tilde{T}_{2}\right\}=0$, and

$$
\left\{\tilde{T}_{2}, \tilde{T}_{1}\right\}=i\left\{\left\{\tilde{T}_{1}, \tilde{T}_{1}\right\}, \tilde{T}_{1}\right\} \equiv 0
$$

due to the Jacobi identity. Thus, the modified constraints $\tilde{T}_{1}=0$ and $\tilde{T}_{2}=0$ are again first-class constraints.

\section{Noncommutative Dirac equation}

After quantization the Poisson brackets (25) will fix the commutation (anticommutation) relations between the corresponding operators

$$
\begin{aligned}
& {\left[\hat{x}^{\mu}, \hat{x}^{\nu}\right]=-i \theta \varepsilon^{\mu \nu \rho \sigma} \hat{S}_{\rho \sigma}+\frac{i \theta^{2}}{2} \varepsilon^{\mu \nu \rho \sigma} \hat{W}_{\rho} \hat{p}_{\sigma}} \\
& {\left[\hat{x}^{\mu}, \hat{p}^{\nu}\right]=i g^{\mu \nu}, \quad\left[\hat{\xi}^{\mu}, \hat{\xi}^{\nu}\right]_{+}=g^{\mu \nu}, \quad\left[\hat{\xi}^{5}, \hat{\xi}^{5}\right]_{+}=-1} \\
& {\left[\hat{x}^{\mu}, \hat{\xi}^{\nu}\right]=-i \theta \varepsilon^{\mu \nu \rho \sigma} \hat{\xi}_{\rho} \hat{p}_{\sigma} .}
\end{aligned}
$$


The operators $\hat{\xi}^{\mu}, \hat{\xi}^{5}$ are generators of the Clifford algebra $C_{5}$. Its representation is four dimensional and is given by the Dirac matrices:

$$
\hat{\xi}^{\mu}=i \gamma^{5} \gamma^{\mu} / \sqrt{2}, \quad \hat{\xi}^{5}=i \gamma^{5} / \sqrt{2} .
$$

The representation of the operators of noncommutative coordinates $\hat{x}^{\mu}$ and momenta $\hat{p}^{\mu}$ is

$$
\hat{x}^{\mu}=x^{\mu} \mathbf{I}-\frac{i \theta}{2} \varepsilon^{\mu \nu \alpha \beta} \hat{S}_{\alpha \beta} \partial_{\nu}, \quad \hat{p}_{\mu}=-i \partial_{\mu} \mathbf{I},
$$

where $\mathbf{I}$ is $4 \times 4$ unit matrix, and

$$
\hat{S}_{\alpha \beta}=-\frac{i}{2}\left(\hat{\xi}_{\alpha} \hat{\xi}_{\beta}-\hat{\xi}_{\beta} \hat{\xi}_{\alpha}\right)=-\frac{1}{4}\left(\gamma_{\alpha} \gamma_{\beta}-\gamma_{\beta} \gamma_{\alpha}\right)=\frac{i}{2} \sigma_{\alpha \beta}
$$

The first equation of (31) is the analog of the Bopp shift; it can be also represented as

$$
\hat{x}^{\mu}=x^{\mu} \mathbf{I}-\frac{i \theta}{2} \gamma^{5} \sigma^{\mu \nu} \partial_{\nu}
$$

Following [21] we define the star product through the Weyl symmetrically ordered operator product as

$$
\mathcal{W}(f \star g)=\mathcal{W}(f) \cdot \mathcal{W}(g)
$$

where

$$
\mathcal{W}(f)=\hat{f}(\hat{x})=\int \frac{d^{4} k}{(2 \pi)^{4}} \tilde{f}(k) e^{-i k_{\mu} \hat{x}^{\mu}},
$$

and $\tilde{f}(p)$ is the Fourier transform of $f$. This product is associative due to the associativity of the operator products. Since,

$$
\left[-i k_{\alpha} x^{\alpha}, k_{\mu} \theta \gamma^{5} \sigma^{\mu \nu} \partial_{\nu} / 2\right]=0
$$

the exponential in the integral (35) can be represented as

$$
e^{-i k_{\mu} \hat{x}^{\mu}}=e^{-i k_{\mu} x^{\mu}} e^{k_{\mu} \theta \gamma^{5} \sigma^{\mu \nu} \partial_{\nu} / 2}
$$

So,

$$
\mathcal{W}(f) \cdot 1=f(x)
$$

the result of the action of the polydifferential operator on a constant is a function. The equations (34) and (37) yield the following formula

$$
(f \star g)(x)=\mathcal{W}(f) g(x)=\hat{f}(\hat{x}) g(x),
$$

where the right hand side means an action of a polydifferential operator on a function. The equation (38) can be written as

$$
\begin{aligned}
& \int \frac{d^{4} k}{(2 \pi)^{4}} \tilde{f}(k) e^{-i k_{\mu} x^{\mu}} e^{k_{\mu} \theta \gamma^{5} \sigma^{\mu \nu} \partial_{\nu} / 2} g(x) \\
& =f g+\sum_{n=1}^{\infty} \frac{\theta^{n}}{2^{n} n !} \int \frac{d^{4} k}{(2 \pi)^{4}} \tilde{f}(k) e^{-i k_{\mu} x^{\mu}}\left(-i k_{\mu_{1}}\right) \ldots\left(-i k_{\mu_{n}}\right) \gamma^{5} \sigma^{\mu_{1} \nu_{1}} \partial_{\nu_{1}} \ldots \gamma^{5} \sigma^{\mu_{n} \nu_{n}} \partial_{\nu_{n}} g(x) .
\end{aligned}
$$


Finally we obtain the expression for the star product as

$$
f \star g=f \exp \left\{\frac{i \theta}{2} \overleftarrow{\partial_{\mu}} \gamma^{5} \sigma^{\mu \nu} \overrightarrow{\partial_{\nu}}\right\} g
$$

The first-class constraints (26), (27) are converted into conditions on the physical states

$$
\hat{T}_{1} \psi=0, \quad \hat{T}_{2} \psi=0,
$$

where some ordering should be specified. We choose the Weyl ordering. Using the representation (30)-(33) of the algebra (29) one writes the first equation of (40) as

$$
\left[i \gamma^{\mu}\left(\partial_{\mu}+i e A_{\mu}\left(x^{\mu} \mathbf{I}-\frac{i \theta}{2} \gamma^{5} \sigma^{\mu \nu} \partial_{\nu}\right)\right)-m\right] \psi=0 .
$$

Taking into account the definition of the star product (39), the above equation can be represented in the form

$$
\left[i \gamma^{\mu}\left(\partial_{\mu}+i e A_{\mu}(x)\right)-m\right] \star \psi=0 .
$$

We call this equation as noncommutative Dirac equation. In contrast to the case of canonical noncommutativity, it is a relativistic equation (in the sense of special relativity), covariant under the Lorentz transformation

$$
x^{\mu} \rightarrow x^{\prime \mu}=\Lambda_{\nu}^{\mu} x^{\nu}, \quad \psi \rightarrow \psi^{\prime}\left(x^{\prime}\right)=S(\Lambda) \psi(x), A^{\mu} \rightarrow A^{\prime \mu}\left(x^{\prime}\right)=\Lambda_{\nu}^{\mu} A^{\nu}(x),
$$

where $S(\Lambda)$ belongs to the usual spinor representation of the Lorentz group. This assertion follows by a direct use of the identities

$$
S^{-1} \gamma^{\mu} S=\Lambda_{\alpha}^{\mu} \gamma^{\alpha}, \quad S^{-1} \sigma^{\mu \nu} S=\Lambda_{\alpha}^{\mu} \Lambda_{\beta}^{\nu} \sigma^{\alpha \beta} .
$$

The second equation of (40) is a consequence of the first one, since $\hat{T}_{2}=\left(\hat{T}_{1}\right)^{2}$.

Note, that a quasiclassical approximation in the spin degrees of freedom (e.g., a partial quantization of bosonic coordinates only) leads to the noncommutativity with bifermionic NC parameter [22]:

$$
x^{\mu} \star x^{\nu}-x^{\nu} \star x^{\mu}=i \Theta^{\mu \nu}, \quad \Theta^{\mu \nu}=i \theta \varepsilon^{\mu \nu \rho \sigma} \xi^{\rho} \xi^{\sigma} / 2 .
$$

Similar constructions also appeared in the context of nonanticommutative superspace [23]. Nilpotent noncommutativity can improve the renormalizability properties of noncommutative theories, [24]. 


\section{Conclusions}

In the present paper we have derived a model of noncommutativity with mixed spatial and spin degrees of freedom. For that we have constructed a consistent deformation of the Berezin-Marinov pseudoclassical formulation of spin particle. In the nonrelativistic case the deformed coordinates are the sum of the initially commutative coordinates and the spin angular momentum, $\tilde{x}^{i}=x^{i}+\theta S^{i}$. The Poisson brackets between the deformed coordinates are proportional to the spin angular momentum, which leads to the spin noncommutativity after quantization. In the relativistic case the deformed coordinates are the sum of the commutative coordinates and the Pauli-Lubanski vector, $\tilde{x}^{\mu}=x^{\mu}+\theta W^{\mu}$.

Also we have obtained the modified Pauli equation (in the nonrelativistic case) and the noncommutative Dirac equation (in the relativistic case), describing spin half particle in an external electromagnetic field in the presence of the spin noncommutativity. Nonlocality in our model depends on the spin of the particle.

We stress that the noncommutative Dirac equation (42) is covariant under Lorentz transformations. Therefore, it can be used as a basis for the construction of a consistent relativistic noncommutative field theory. The next step in this way is to introduce the trace functional on the algebra of the star product (40) and to construct corresponding action functional for the noncommutative Dirac field. Also, stell in the context of quantum mechanics, it would be interesting to study phenomenological effects caused by the spin noncommutativity on the examples of exact solvable QM models like Hydrogen atom, Landau problem, AharonovBohm effect, etc.

\section{Acknowledgements}

V.G.K. acknowledges FAPESP for support. M.G. and A.J.S. thank FAPESP and CNPq for partial support.

\section{References}

[1] R. Peierls, Z.Phys. 80 (1933) 763.

[2] N. Seiberg and E. Witten, JHEP 9909 (1999) 032.

[3] M. Douglas, N. Nekrasov, Rev.Mod.Phys.73 (2001) 977-1029; R. Szabo, Phys.Rept.378 (2003) 207-299.

[4] C. Duval, P.A. Horvathy, Phys.Lett.B479(2000)284; M. Chaichian, M.M. Sheikh-Jabbari and A. Tureanu, Phys.Rev.Lett. 86(2001)2716; J. Gamboa, M. Loewe and J.C. Rojas, Phys.Rev.D64 (2001)067901; V.P. Nair, A.P. Polychronakos, Phys.Lett.B505(2001)267; S. Bellucci, A. 
Nersessian, C. Sochichiu, Phys.Lett.B522(2001)345; P.A. Horvathy, M.S. Plyushchay, JHEP06(2002)033; A.F. Ferrari, M. Gomes, C.A. Stechhahn, Phys.Rev.D76(2007)085008; P.G. Castro, B. Chakraborty, F. Toppan, J.Math.Phys.49(2008)082106.

[5] M. Gomes, V.G. Kupriyanov, Phys.Rev.D79 (2009) 125011.

[6] M. Gomes, V.G. Kupriyanov, A.J. da Silva, Dynamical noncommutativity, arXiv:0908.2963 [hep-th].

[7] H. Falomir, J. Gamboa, J. Lopez-Sarrion, F. Mendez, P.A.G. Pisani, Phys.Lett.B680 (2009) 384-386.

[8] J. Gamboa, F. Mendez, Bose-Einstein condensation theory for any integer spin: approach based in noncommutative quantum mechanics, arXiv:0912.2645 [hep-th].

[9] R. Jackiw, V.P. Nair, Phys.Lett.B480(2000)237.

[10] J. Lukierski, P.C. Stichel., W.J. Zakrzewski, Annals Phys.260 (1997) 224249.

[11] C. Duval, P. Horvathy, J. Phys. A 34 (2001) 10097.

[12] A.A. Deriglazov, Phys.Lett. B555 (2003) 83; JHEP 0303 (2003) 021.

[13] C. Acatrinei, JHEP 09(2001)007.

[14] D.M. Gitman, V.G. Kupriyanov, Eur.Phys.J. C 54 (2008) 325.

[15] F.S. Bemfica, H.O. Girotti, Phys.Rev.D77 (2008) 027704, Phys.Rev.D79 (2009) 125024;

[16] D.M. Gitman, V.G. Kupriyanov, J.Math.Phys. 51 (2010) 022905.

[17] F. A. Berezin and M. S. Marinov, Ann. Phys. 104 (1977) 336.

[18] A. Barducci, R. Casalbuoni, L. Lusanna, Nuovo Cimento 35A (1976) 377.

[19] P.A. Horvathy, Acta Phys.Pol. B34 (2003) 2611.

[20] D.M. Gitman and V.G. Kupriyanov, Eur.Phys.J.C 50 (2007) 691-700.

[21] V.G. Kupriyanov, D.V. Vassilevich, Eur.Phys.J.C. 58 (2008) 627-637.

[22] D.M. Gitman, D.V. Vassilevich, Mod.Phys.Lett.A23 (2008) 887-893.

[23] N. Seiberg, JHEP 0306 (2003) 010.

[24] R. Fresneda, D.M. Gitman, D.V. Vassilevich, Phys.Rev.D78 (2008) 025004. 